Clinical Medicine

Oral

Abstract ID: 72

\title{
The functional outcome and quality of life following open diaphyseal tibial fractures
}

Nazri Mohd Yusof | Mohd Shahidan Noor Rahin

Kulliyyah of Medicine, International Islamic University Malaysia

Introduction: The clinical outcome of open tibia fracture been extensively studied in many institutions. However, the functional outcome and quality of life has not been widely studied. The aim of this study is to evaluate the functional outcome and quality of life for patients with open tibia fracture that were treated in Hospital Tengku Ampuan Afzan. Methods: Between 2009 and 2010, we treated 33 patients with open diaphyseal tibial fractures with a minimum period of one year follow up. There were 31 males and 2 females; the mean age of 29 years old (range: 10 to 60). Four patients sustained open fracture grade I, 13 grade II, 12 grade IIIA and 4 with grade IIIB. All patients who fulfill the inclusion criteria were called for interview in the clinic by the investigator. The outcome that were analyzed include health related quality of life (SF36) and Lower Extremity Functional Score (LEFS). Results: All except one fracture achieve union. One patient with grade IIIB open fracture developed infected non union. Quality of life is satisfactory in all domains. The functional outcome score was significantly reduced in grade IIIB (48.5) as compare to other grade (grade I 74.2, grade II 70.4, grade IIIA 74.1). Conclusions: The quality of life and the functional outcome worsened with increasing severity of fracture.

KEYWORDS: open fracture, tibia, quality of life, functional outcome 